\title{
HLA-DRB1* Allele Frequencies in Pediatric, Adolescent and Adult-Onset Multiple Sclerosis Patients, in a Hellenic Sample. Evidence for New and Established Associations
}

\author{
Maria C Anagnostouli ${ }^{1,2 *}$, Argyro Manouseli ${ }^{1,3}$, Artemios K Artemiadis ${ }^{1}$, Serafeim Katsavos ${ }^{1}$, Constantina Fillipopoulou ${ }^{1,3}$, Sotirios Youroukos $^{1}$, Spyros \\ Efthimiopoulos ${ }^{3}$ and llias Doxiadis ${ }^{4}$ \\ ${ }^{1}$ Immunogenetics Laboratory, 1st Department of Neurology, Aeginition Hospital, Medical School of National and Kapodistrian University of Athens, Athens, Greece \\ 21st Department of Neurology, Aeginition Hospital, Medical School of National and Kapodistrian University of Athens, Athens, Greece \\ ${ }^{3}$ Department of Biology, Division of Animal and Human Physiology of National and Kapodistrian University of Athens, Athens, Greece \\ ${ }^{4}$ Department of Immunohaematology and Blood Transfusion, University Hospital, Leiden, The Netherlands \\ ${ }^{*}$ Corresponding author: Maria C Anagnostouli, Assistant Professor of Neurology and Director of Immunogenetics Laboratory, 1st Department of Neurology, Aeginition \\ Hospital, National and Kapodistrian University of Athens, 72-74 Vas. Sophias Avenue, 11528, Greece, Tel: +30 2107289165 ; Fax: +30 210 685 2913; E-Mail:
}

managnost@med.uoa.gr

Received date: Apr 1, 2014, Accepted date: May 22, 2014, Published date: May 26, 2014

Copyright: (c) 2014 Anagnostouli MC, et al. This is an open-access article distributed under the terms of the Creative Commons Attribution License, which permits unrestricted use, distribution, and reproduction in any medium, provided the original author and source are credited.

\begin{abstract}
Studies in many populations consistently have showed that the human leukocyte antigens (HLA) and especially the DRB1*15 allele has by far the strongest genetic association with multiple sclerosis (MS). The aim of this study was to investigate the role of HLA-DRB1 ${ }^{*}$ alleles in MS risk/resistance and onset. A sample of 165 Hellenic MS patients (18 with pediatric-, 24 with adolescent- and 123 with adult-onset MS) and 107 healthy volunteers were examined with molecular techniques. Comparisons were made according to the Benjamini-Yekutieli method for $p$ value correction. Both adult-onset MS patients and early-onset MS patients (age at onset below 20 years-old) had a significantly higher frequency of the DRB1* 15 allele and a significantly lower frequency of the DRB1 11 allele compared to controls. For the early-onset vs. healthy group comparison, subgroup analyses revealed that both the pediatric- and the adolescent-onset MS groups contributed to the aforementioned DRB1*15 significant difference, while the DRB1*11 difference was ascribed solely to the adolescent-MS onset vs. healthy group comparison. Within MS patients comparisons revealed that early-onset MS patients had a tendency for higher DRB1*03 allele and a lower DRB1*16 allele frequency frequencies compared to adult-onset MS patients, although both non-significant. Notably, pediatric-onset MS patients showed complete absence of the DRB1 ${ }^{*} 16$ allele, along with a non-significant tendency for higher DRB1 ${ }^{*} 15$ allele frequency relative to the adult-onset group. Finally, the adolescent-onset MS group was presented with a lower DRB1 ${ }^{*} 11$ allele frequencies compared both to the pediatric- and the adult-onset MS group. Our findings confirm previous studies on the role of HLA-DRB1 ${ }^{*}$ in MS. New findings that need to be confirmed by further studies are the pathogenetic role of DRB $1^{*} 03$ for early-onset MS and the putative protective role of the DRB1*16 allele in the pediatric-onset MS.
\end{abstract}

Keywords Multiple sclerosis; HLA; Genetics; Immunogenetics; Pediatric; Adolescence; Hellenic population

\section{Introduction}

Multiple sclerosis (MS) is a chronic autoimmune demyelinating disease of the central nervous system (CNS) of unknown origin. Both environmental and genetic factors have been implicated in the pathogenesis of the disease leading to the well-known consideration of MS as a complex multi-factorial disease entity [1]. Concerning genetics, linkage studies in many populations consistently have showed that the human leukocyte antigens (HLA), products of the Major Histocompatibility Complex (MHC) on chromosome 6p21.3 are linked to MS [1,2]. MHC represents a cluster of highly polymorphic genes, including the HLA-Class I, II and III genes that encode proteins which serve antigen presentation to T-lymphocytes. Different auto antigens have different binding capacities to HLA molecule, explaining ostensibly the predisposing role of HLA in autoimmune diseases.
Historically, HLA-A and -B (HLA class I) alleles were the first to be associated with adult-onset MS, with HLA class II (such as HLA-DR2 and DQw6) identified in later studies [3,4]. In a recent collaborative European study, DRB1*1501 (split of DR2) had the strongest association with MS, along with $\mathrm{DRB1} 1^{\star} 0301$ and $\mathrm{DRB1} 1^{\star} 1301$, while HLA-A ${ }^{\star} 0201$ has been found to confer protection against MS [5]. Studies in other populations reveal different candidate alleles such as $\mathrm{DPA}^{*} 02, \mathrm{DPB} 1^{\star} 05, \mathrm{DRB} 1^{\star} 0403$ and $\mathrm{DRB} 1{ }^{*} 08$ [6-9].

Notably, there is corroborating evidence outlining the prominent role of HLA-DRB1* in adult-onset MS. First of all, HLA-DRB1 has been found to be regulated by vitamin $\mathrm{D}$, which is associated with the risk for MS [10]. The presence of $\mathrm{DRB1}^{\star} 15$ has also been associated with more disability, more severe spinal cord pathology, positive oligoclonal bands, more potent humoral responses to Epstein-Barr virus, female susceptibility to the disease and earlier onset [11-15]. On the other hand, in some populations, $\mathrm{DRB} 1^{\star} 01, \mathrm{DRB} 1^{\star} 07, \mathrm{DRB} 1^{\star} 11$ and DRB1 $1^{\star} 14$ appeared to provide protection from the disease [16].

Patients with early-onset MS (before 18 years old) account for the 3.5- $5 \%$ of the general pool of the MS patients [17]. DRB $1^{\star} 15$ association with childhood and or earlier onset of MS has been 
Citation: Anagnostouli MC, Manouseli A, Artemiadis AK, Katsavos S, Fillipopoulou C et al. (2014) HLA-DRB1* Allele Frequencies in Pediatric, Adolescent and Adult-Onset Multiple Sclerosis Patients, in a Hellenic Sample. Evidence for New and Established Associations. J Mult Scler 1: 104. doi:10.4172/jmso.1000104

Page 2 of 8

attested by studies $[18,19]$. Moreover, $\mathrm{DRB}^{\star} 04$ combined with $\mathrm{DRB1}^{\star} 15$ has been linked with earlier onset of the disease but it may delay age at onset when combined with $\mathrm{DRB} 1^{\star} 0801[20]$. In a Korean population, close linkage of DRB $3^{\star} 02, \mathrm{DRB} 1^{\star} 13$ and $\mathrm{DQB} 1^{\star} 03$ was also associated with the risk of childhood MS [21]. Although the scarcity of MS during adolescence could be attributed to the antiinflammatory and/or neuroprotective role of both androgens and estrogens which are found increased during this age-period, the interplay between HLA and these hormones and its consequences on autoimmunity have been poorly studied in MS $[22,23]$.

In a previous study presenting a three generation Hellenic familial case of bipolar disorder in which there was comorbidity with MS, by Bozikas et al. [24] it was the first time that a sample of 87 Hellenic MS patients had been genotyped for HLA in our laboratory and the increased frequency of $\mathrm{DRB} 1^{\star} 1501$ was found and presented, compared to healthy controls. In contrary, in this study, it was also showed that the members of this three generation family (of both juvenile and adulthood patients) carried the DRB1*16 allele [24]. In the second HLA Hellenic study by Kouri et al. [25] DRB1 ${ }^{\star} 1501$, $\mathrm{DQB} 1^{\star} 0602$ and $\mathrm{DQA1}{ }^{\star} 0102$ alleles were more frequent among patients than controls, with the first two detected more frequently in patients with initial symptoms from the brainstem or the cerebellum [25]. The present study expands literature on HLA, by investigating the influence of HLA-DRB1* alleles on disease risk/resistance and age at onset in a Hellenic sample of MS patients, for the first time, with both early-onset (pediatric and adolescent) and adulthood MS, using healthy controls.

\section{Materials and Methods}

\section{Subjects}

A group of 165 Hellenic MS patients, diagnosed according to the McDonald criteria, was studied at the Immunogenetics Laboratory of the 1st Department of Neurology, of Athens National University, between 2002 and 2012 [26]. According to the age at disease onset, three subcategories were recognized: 1 . Two with early-onset MS: a eighteen ( 8 males, 10 females, median EDSS: 3.25, EDSS range: 1.5-7) with pediatric-onset MS (below 16 years old) (median age: 14, range: 9-15 years old), b. twenty-four ( 7 males, 17 females, median EDSS: 3.5 EDSS range: 1-7) with adolescent-onset (between 16 and 19 years old) (median age: 17) and 2. One hundred and twenty-three (46 males, 77 females, median EDSS: 3, EDSS range: 0-8) with adult-onset MS (above 19 years old) (median age: 37.5, range: 21-69 years old). One hundred and seven (48 males and 59 females) Hellenic healthy volunteers, with no history of autoimmune or inflammatory disease were selected from our laboratory records. Each individual gave his/her informed consent and hospital's ethical committee approved this study.

\section{HLA genotyping}

HLA genotyping was performed at the Immunogenetics Laboratory of the 1st Department of Neurology in Aeginition Hospital. High molecular weight DNA was extracted from peripheral blood samples
(8mL peripheral blood in sodium citrate, ACD Vacutainer $\left.{ }^{\oplus}\right)$. HLA class II $\left(\mathrm{DRB1}^{*}\right)$ frequencies were determined by molecular techniques, in all subjects, for all the specificities included in the HLA Nomenclature of 2012 (we present only the first four numbers of each allele) [27]. After DNA extraction (QIAGEN Maxi Kit), the polymorphic second exon of the HLA-DRB1*gene was amplified and the products were genotyped using PCR-SSO analysis for class II (BioRad-Elpha, High resolution). The technique we have used to perform HLA-DRB1* genotyping was an SSO (Sequence-Specific Oligonucleotide), which results in final absorptions of an Elisa format, which cannot be stored in another way, except for an Elisa sheet. The results of the absorptions are put in an electronic system especially created for this reason (Bio-Rad-Elpha), which finally converts the positive Elisa positions (higher absorptions than the reference) to HLA-DRB1* alleles. We have stored all the Elisa-sheets in our personal computer, along with the electronic results for HLA-DRB1 ${ }^{\star}$ for all our samples [28].

\section{Statistical analyses}

Frequencies of the DRB1* alleles were compared across groups using two-sample chi-square test. Groups according to disease onset were: before 19 years old $(\leq 19)$ or early-onset MS, after 19 years old $(>19)$ or adult-onset MS, before 16 years old $(\leq 15)$ or pediatric-onset MS and 16-19 years old or adolescent-onset MS. P value correction was made according to the Benjamini-Yekutieli method (or B-Y) according to the formula $\mathrm{p}(\mathrm{B}-\mathrm{Y})=\mathrm{a} /(\Sigma 1 / \mathrm{i})$ where $i$ denotes the number of comparisons and $a=0.05$. As such, the level of significance for 11 comparisons (corresponding to 11 alleles) was 0.017 and for 12 comparisons 0.016. This method has been proposed to be less conservative than the Bonferroni especially in case of multiple comparisons, reducing type II error [29]. Statistical analyses were done using SPSS 18.0 software (SPSS Inc., Chicago, IL, USA).

\section{Results}

Tables 1,2,3 and 4 represent comparisons of MS patients with earlyonset disease, pediatric-onset MS, adolescent-onset MS and adultonset MS versus healthy individuals. Patients with early-onset MS had significantly higher frequencies of the $\mathrm{DRB} 1^{\star} 15$ allele [OR 2.653, (1.219-5.774), $\mathrm{p}=0.016$ ] and significantly lower of the $\mathrm{DRB} 1^{\star} 11$ allele [OR $0.448,(0.236-0.853), \mathrm{p}=0.014]$. There was also a non-statistical significant tendency for higher $\mathrm{DRB}^{\star} 03$ allele frequency in MS patients of this age. The DRB1 ${ }^{\star} 16$ allele was found significantly absent in the pediatric group $(p=0.011)$, along with statistically nonsignificant tendencies for higher frequency of both the $\mathrm{DRB} 1^{*} 03$ and the DRB $1^{*} 15$ allele. DRB $1^{*} 11$ allele was significantly lower in the adolescent group [OR 0.204, $(0.07-0.591), \mathrm{p}=0.01$ ], along with statistically non-significant tendencies for higher frequency of both the $\mathrm{DRB}^{\star} 03$ and the $\mathrm{DRB} 1^{\star} 15$ allele. Adult-onset MS (above 20 years old) was characterized by significantly higher $\mathrm{DRB1}^{\star} 15$ allele frequency $[\mathrm{OR}=2.653,(1.423-4.946), \mathrm{p}=0.003]$ and significantly lower $\mathrm{DRB}^{\star}{ }^{\star} 11$ allele frequency $[\mathrm{OR}=0.462,(0.297-0.718), \mathrm{p}=0.001]$. 
Citation: Anagnostouli MC, Manouseli A, Artemiadis AK, Katsavos S, Fillipopoulou C et al. (2014) HLA-DRB1* Allele Frequencies in Pediatric, Adolescent and Adult-Onset Multiple Sclerosis Patients, in a Hellenic Sample. Evidence for New and Established Associations. J Mult Scler 1: 104. doi:10.4172/jmso.1000104

Page 3 of 8

\begin{tabular}{|c|c|c|c|c|c|c|c|c|c|c|}
\hline \multirow{2}{*}{ HLA-DRB1* } & \multirow{2}{*}{$\begin{array}{l}\begin{array}{l}\text { Early-ons } \\
\text { alleles) }\end{array} \\
\text { Positive }\end{array}$} & \multicolumn{2}{|c|}{ Multiple Sclerosis $\quad(84$} & \multicolumn{3}{|c|}{ Healthy Individuals (214 alleles) } & \multicolumn{4}{|c|}{ Fisher Exact test } \\
\hline & & Negative & $\%$ & Positive & Negative & $\%$ & OR & $\begin{array}{l}\text { Lower } \\
\text { Limit }\end{array}$ & $\begin{array}{l}\text { Upper } \\
\text { Limit }\end{array}$ & $p$ value \\
\hline DRB $1 * 01$ & 5 & 79 & 6 & 12 & 202 & 6 & 1.065 & 0.364 & 3.122 & 1 \\
\hline $\mathrm{DRB} 1{ }^{*} 03$ & 15 & 69 & 18 & 20 & 194 & 9 & 2.109 & 1.023 & 4.348 & 0.047 \\
\hline $\mathrm{DRB} 1^{*} 04$ & 10 & 74 & 12 & 17 & 197 & 8 & 1.566 & 0.686 & 3.575 & 0.369 \\
\hline $\mathrm{DRB} 1^{*} 07$ & 3 & 81 & 4 & 7 & 207 & 3 & 1.095 & 0.276 & 4.339 & 1 \\
\hline $\mathrm{DRB} 1^{*} 08$ & 0 & 0 & 0 & 5 & 209 & 2 & $\mathrm{NE}$ & $\mathrm{NE}$ & NE & 0.327 \\
\hline DRB $1 * 10$ & 2 & 82 & 2 & 6 & 208 & 3 & 0.846 & 0.167 & 4.275 & 1 \\
\hline DRB1*11 & 14 & 70 & 17 & 66 & 148 & 31 & 0.448 & 0.236 & 0.853 & $0.014^{*}$ \\
\hline DRB1*12 & 1 & 83 & 1 & 5 & 209 & 2 & 0.504 & 0.058 & 4.376 & 1 \\
\hline DRB1*13 & 7 & 77 & 8 & 21 & 193 & 1 & 0.835 & 0.341 & 2.045 & 0.827 \\
\hline DRB1*14 & 5 & 79 & 6 & 11 & 203 & 5 & 1.168 & 0.393 & 3.469 & 0.779 \\
\hline DRB1*15 & 14 & 70 & 17 & 15 & 199 & 7 & 2.653 & 1.219 & 5.774 & $0.016^{*}$ \\
\hline DRB1*16 & 8 & 76 & 10 & 29 & 185 & 14 & 0.672 & 0.294 & 1.535 & 0.436 \\
\hline
\end{tabular}

Table 1: HLA-DRB1* frequencies and comparisons between patients with early-onset multiple sclerosis $(\mathrm{N}=42)$ and healthy individuals $(\mathrm{N}=107)$.

\begin{tabular}{|c|c|c|c|c|c|c|c|c|c|c|}
\hline \multirow{2}{*}{ HLA-DRB1* } & \multicolumn{3}{|c|}{$\begin{array}{l}\text { Pediatric-onset Multiple Sclerosis ( } 36 \\
\text { alleles) }\end{array}$} & \multicolumn{3}{|c|}{ Healthy Individuals (214 alleles) } & \multicolumn{4}{|c|}{ Fisher Exact test } \\
\hline & Positive & Negative & $\%$ & Positive & Negative & $\%$ & OR & Lower Limit & Upper Limit & $p$ value \\
\hline $\mathrm{DRB} 1{ }^{*} 01$ & 1 & 35 & 3 & 12 & 202 & 6 & 0.481 & 0.061 & 3.816 & 0.699 \\
\hline DRB $1 * 03$ & 7 & 29 & 19 & 20 & 194 & 9 & 2.341 & 0.91 & 6.024 & 0.083 \\
\hline $\mathrm{DRB} 1 * 04$ & 5 & 31 & 14 & 17 & 197 & 8 & 1.869 & 0.643 & 5.431 & 0.334 \\
\hline DRB1*07 & 2 & 34 & 6 & 7 & 207 & 3 & 1.739 & 0.347 & 8.727 & 0.621 \\
\hline DRB1*08 & 0 & 0 & 0 & 5 & 209 & 2 & $\mathrm{NE}$ & $\mathrm{NE}$ & $\mathrm{NE}$ & 1 \\
\hline DRB1*10 & 1 & 35 & 3 & 6 & 208 & 3 & 0.99 & 0.116 & 8.478 & 1 \\
\hline DRB1*11 & 10 & 26 & 28 & 66 & 148 & 31 & 0.862 & 0.393 & 1.891 & 0.845 \\
\hline DRB1*12 & 0 & 36 & 0 & 5 & 209 & 2 & $\mathrm{NE}$ & $\mathrm{NE}$ & $\mathrm{NE}$ & 1 \\
\hline DRB1*13 & 3 & 33 & 8 & 21 & 193 & 10 & 0.835 & 0.236 & 2.96 & 1 \\
\hline DRB1*14 & 1 & 35 & 3 & 11 & 203 & 5 & 0.527 & 0.066 & 4.213 & 1 \\
\hline DRB1*15 & 6 & 30 & 17 & 15 & 199 & 7 & 2.653 & 0.955 & 7.37 & 0.095 \\
\hline DRB1*16 & 0 & 36 & 0 & 29 & 185 & 14 & $\mathrm{NE}$ & $\mathrm{NE}$ & $\mathrm{NE}$ & $0.011^{*}$ \\
\hline
\end{tabular}

Table 2: HLA-DRB1* frequencies and comparisons between patients with pediatric-onset multiple sclerosis $(\mathrm{N}=18)$ and healthy individuals $(\mathrm{N}=107)$. 
Citation: Anagnostouli MC, Manouseli A, Artemiadis AK, Katsavos S, Fillipopoulou C et al. (2014) HLA-DRB1* Allele Frequencies in Pediatric, Adolescent and Adult-Onset Multiple Sclerosis Patients, in a Hellenic Sample. Evidence for New and Established Associations. J Mult Scler 1: 104. doi:10.4172/jmso.1000104

Page 4 of 8

\begin{tabular}{|c|c|c|c|c|c|c|c|c|c|c|}
\hline \multirow{2}{*}{ HLA-DRB1* } & \multicolumn{3}{|c|}{$\begin{array}{l}\text { Adolescent-onset Multiple Sclerosis (48 } \\
\text { alleles) }\end{array}$} & \multicolumn{3}{|c|}{ Healthy Individuals (214 alleles) } & \multicolumn{4}{|c|}{ Fisher Exact test } \\
\hline & Positive & Negative & $\%$ & Positive & Negative & $\%$ & OR & $\begin{array}{l}\text { Lower } \\
\text { Limit }\end{array}$ & $\begin{array}{l}\text { Upper } \\
\text { Limit }\end{array}$ & $\mathrm{p}$ value \\
\hline DRB $1 * 01$ & 4 & 44 & 8 & 12 & 202 & 6 & 1.53 & 0.471 & 4.968 & 0.504 \\
\hline DRB1*03 & 8 & 40 & 17 & 20 & 194 & 9 & 1.94 & 0.798 & 4.714 & 0.192 \\
\hline DRB1*04 & 5 & 43 & 10 & 17 & 197 & 8 & 1.347 & 0.471 & 3.852 & 0.568 \\
\hline $\mathrm{DRB} 1{ }^{*} 07$ & 1 & 47 & 2 & 7 & 207 & 3 & 0.629 & 0.076 & 5.237 & 1 \\
\hline DRB $1 * 08$ & 0 & 0 & 0 & 5 & 209 & 2 & NE & $\mathrm{NE}$ & NE & 0.588 \\
\hline DRB1*10 & 1 & 47 & 2 & 6 & 208 & 3 & 0.738 & 0.087 & 6.272 & 1 \\
\hline DRB1*11 & 4 & 44 & 8 & 66 & 148 & 31 & 0.204 & 0.07 & 0.591 & $0.001^{*}$ \\
\hline DRB $1 * 12$ & 1 & 47 & 2 & 5 & 209 & 2 & 0.889 & 0.102 & 7.791 & 1 \\
\hline DRB1*13 & 4 & 44 & 8 & 21 & 193 & 10 & 0.835 & 0.273 & 2.556 & 1 \\
\hline DRB1*14 & 4 & 44 & 8 & 11 & 203 & 5 & 1.678 & 0.51 & 5.514 & 0.488 \\
\hline DRB1*15 & 8 & 40 & 17 & 15 & 199 & 7 & 2.653 & 1.054 & 6.677 & 0.046 \\
\hline DRB1*16 & 8 & 40 & 17 & 29 & 185 & 14 & 1.276 & 0.543 & 2.997 & 0.646 \\
\hline
\end{tabular}

Table 3: HLA-DRB1* frequencies and comparisons between patients with adolescent-onset multiple sclerosis $(\mathrm{N}=24)$ and healthy individuals $(\mathrm{N}=107)$.

\begin{tabular}{|c|c|c|c|c|c|c|c|c|c|c|}
\hline \multirow{2}{*}{ HLA-DRB1* } & \multirow{2}{*}{$\begin{array}{l}\begin{array}{l}\text { Adult-onse } \\
\text { alleles) }\end{array} \\
\text { Positive }\end{array}$} & \multicolumn{2}{|c|}{ Multiple Sclerosis $\quad(246$} & \multicolumn{3}{|c|}{ Healthy Individuals (214 alleles) } & \multicolumn{4}{|c|}{ Fisher Exact test } \\
\hline & & Negative & $\%$ & Positive & Negative & $\%$ & OR & $\begin{array}{l}\text { Lower } \\
\text { Limit }\end{array}$ & $\begin{array}{l}\text { Upper } \\
\text { Limit }\end{array}$ & $\mathrm{p}$ value \\
\hline DRB $1 * 01$ & 19 & 227 & 7 & 12 & 202 & 6 & 1.409 & 0.667 & 2.974 & 0.474 \\
\hline $\mathrm{DRB} 1{ }^{*} 03$ & 22 & 224 & 9 & 20 & 194 & 9 & 0.953 & 0.505 & 1.798 & 1 \\
\hline DRB1*04 & 31 & 215 & 13 & 17 & 197 & 8 & 1.671 & 0.897 & 3.113 & 0.14 \\
\hline $\mathrm{DRB} 1{ }^{*} 07$ & 10 & 236 & 4 & 7 & 207 & 3 & 1.253 & 0.469 & 3.351 & 0.84 \\
\hline $\mathrm{DRB} 1{ }^{*} 08$ & 0 & 0 & 0 & 5 & 209 & 2 & $\mathrm{NE}$ & NE & $\mathrm{NE}$ & 0.05 \\
\hline DRB1*10 & 7 & 239 & 3 & 6 & 208 & 3 & 1.015 & 0.336 & 3.069 & 1 \\
\hline DRB1*11 & 42 & 204 & 17 & 66 & 148 & 31 & 0.462 & 0.297 & 0.718 & $0.001^{*}$ \\
\hline DRB1*12 & 6 & 240 & 2 & 5 & 209 & 2 & 1.045 & 0.314 & 3.474 & 1 \\
\hline DRB1*13 & 16 & 230 & 7 & 21 & 193 & 10 & 0.639 & 0.325 & 1.259 & 0.259 \\
\hline DRB1*14 & 12 & 234 & 5 & 11 & 203 & 5 & 0.946 & 0.409 & 2.191 & 1 \\
\hline DRB1*15 & 41 & 205 & 17 & 15 & 199 & 7 & 2.653 & 1.423 & 4.946 & $0.003^{*}$ \\
\hline DRB1*16 & 40 & 206 & 16 & 29 & 185 & 14 & 1.239 & 0.738 & 2.079 & 0.496 \\
\hline
\end{tabular}

Table 4: HLA-DRB1* frequencies and comparisons between patients with adult-onset multiple sclerosis $(\mathrm{N}=123)$ and healthy individuals $(\mathrm{N}=107)$. 
Citation: Anagnostouli MC, Manouseli A, Artemiadis AK, Katsavos S, Fillipopoulou C et al. (2014) HLA-DRB1* Allele Frequencies in Pediatric, Adolescent and Adult-Onset Multiple Sclerosis Patients, in a Hellenic Sample. Evidence for New and Established Associations. J Mult Scler 1: 104. doi:10.4172/jmso.1000104

Page 5 of 8

\begin{tabular}{|c|c|c|c|c|c|c|c|c|c|c|}
\hline \multirow{2}{*}{$\begin{array}{l}\text { HLA-DRB1* } \\
1\end{array}$} & \multirow{2}{*}{$\begin{array}{l}\text { Early-ons } \\
\text { alleles) }\end{array}$} & \multicolumn{2}{|c|}{ Multiple Sclerosis $(84$} & \multicolumn{3}{|c|}{$\begin{array}{l}\text { Adult-onset Multiple Sclerosis (246 } \\
\text { alleles) }\end{array}$} & \multicolumn{4}{|c|}{ Fisher Exact test } \\
\hline & & Negative & $\%$ & Positive & Negative & $\%$ & OR & Lower Limit & Upper Limit & $p$ value \\
\hline DRB1*01 & 5 & 79 & 6 & 19 & 227 & 8 & 0.756 & 0.273 & 2.092 & 0.808 \\
\hline DRB1*03 & 15 & 69 & 18 & 22 & 224 & 9 & 2.213 & 1.089 & 4.5 & 0.043 \\
\hline $\mathrm{DRB} 1{ }^{*} 04$ & 10 & 74 & 12 & 31 & 215 & 13 & 0.937 & 0.438 & 2.004 & 1 \\
\hline $\mathrm{DRB} 1{ }^{*} 07$ & 3 & 81 & 4 & 10 & 236 & 4 & 0.874 & 0.235 & 3.255 & 1 \\
\hline DRB $1 * 10$ & 2 & 82 & 2 & 7 & 239 & 3 & 0.833 & 0.17 & 4.089 & 1 \\
\hline DRB1*11 & 14 & 70 & 17 & 42 & 204 & 17 & 0.971 & 0.501 & 1.885 & 1 \\
\hline DRB1*12 & 1 & 83 & 1 & 6 & 240 & 2 & 0.482 & 0.057 & 4.062 & 0.683 \\
\hline DRB1*13 & 7 & 77 & 8 & 16 & 230 & 7 & 1.307 & 0.518 & 3.295 & 0.62 \\
\hline DRB1*14 & 5 & 79 & 6 & 12 & 234 & 5 & 1.234 & 0.422 & 3.612 & 0.775 \\
\hline DRB1*15 & 14 & 70 & 17 & 41 & 205 & 17 & 1 & 0.514 & 1.944 & 1 \\
\hline DRB $1 * 16$ & 8 & 76 & 1 & 40 & 206 & 16 & 0.542 & 0.243 & 1.211 & 0.153 \\
\hline
\end{tabular}

Table 5: HLA-DRB1* frequencies and comparisons between patients with multiple sclerosis early-onset ( $\mathrm{N}=42)$ and adult-onset multiple sclerosis $(\mathrm{N}=123)$.

\begin{tabular}{|c|c|c|c|c|c|c|c|c|c|c|}
\hline \multirow{2}{*}{ HLA-DRB1* } & \multicolumn{3}{|c|}{$\begin{array}{l}\text { Pediatric-onset Multiple Sclerosis ( } 36 \\
\text { alleles) }\end{array}$} & \multicolumn{3}{|c|}{$\begin{array}{l}\text { Adult-onset Multiple Sclerosis (246 } \\
\text { alleles) }\end{array}$} & \multicolumn{4}{|c|}{ Fisher Exact test } \\
\hline & Positive & Negative & $\%$ & Positive & Negative & $\%$ & OR & $\begin{array}{l}\text { Lower } \\
\text { Limit }\end{array}$ & $\begin{array}{l}\text { Upper } \\
\text { Limit }\end{array}$ & $p$ value \\
\hline DRB $1 * 01$ & 1 & 35 & 3 & 19 & 227 & 8 & 0.341 & 0.044 & 2.631 & 0.487 \\
\hline DRB $1 * 03$ & 7 & 29 & 19 & 22 & 224 & 9 & 2.458 & 0.965 & 6.256 & 0.073 \\
\hline DRB 1*04 & 5 & 31 & 14 & 31 & 215 & 13 & 1.119 & 0.405 & 3.092 & 0.791 \\
\hline DRB $1 * 07$ & 2 & 34 & 6 & 10 & 236 & 4 & 1.388 & 0.292 & 6.608 & 0.655 \\
\hline DRB1*10 & 1 & 35 & 3 & 7 & 239 & 3 & 0.976 & 0.116 & 8.169 & 1 \\
\hline DRB1*11 & 10 & 26 & 28 & 42 & 204 & 17 & 1.868 & 0.838 & 4.163 & 0.164 \\
\hline DRB1*12 & 0 & 36 & 0 & 6 & 240 & 2 & $\mathrm{NE}$ & NE & NE & 1 \\
\hline DRB1*13 & 3 & 33 & 8 & 16 & 230 & 7 & 1.307 & 0.361 & 4.728 & 0.719 \\
\hline DRB1*14 & 1 & 35 & 3 & 12 & 234 & 5 & 0.557 & 0.07 & 4.418 & 1 \\
\hline DRB1*15 & 6 & 30 & 17 & 41 & 205 & 17 & 1 & 0.391 & 2.556 & 1 \\
\hline DRB1*16 & 0 & 36 & 0 & 20 & 206 & 16 & $\mathrm{NE}$ & NE & $\mathrm{NE}$ & $0.004^{*}$ \\
\hline
\end{tabular}

Table 6: HLA-DRB1* frequencies and comparisons between patients with pediatric-onset $(\mathrm{N}=18)$ and adult-onset multiple sclerosis $(\mathrm{N}=123)$. 
Citation: Anagnostouli MC, Manouseli A, Artemiadis AK, Katsavos S, Fillipopoulou C et al. (2014) HLA-DRB1* Allele Frequencies in Pediatric, Adolescent and Adult-Onset Multiple Sclerosis Patients, in a Hellenic Sample. Evidence for New and Established Associations. J Mult Scler 1: 104. doi:10.4172/jmso.1000104

Page 6 of 8

\begin{tabular}{|c|c|c|c|c|c|c|c|c|c|c|}
\hline \multirow{2}{*}{ HLA-DRB1* } & \multicolumn{3}{|c|}{$\begin{array}{l}\text { Pediatric-onset Multiple Sclerosis ( } 36 \\
\text { alleles) }\end{array}$} & \multicolumn{3}{|c|}{$\begin{array}{l}\text { Adolescent-Onset Multiple Sclerosis (48 } \\
\text { alleles) }\end{array}$} & \multicolumn{4}{|c|}{ Fisher Exact test } \\
\hline & Positive & Negative & $\%$ & Positive & Negative & $\%$ & OR & Lower Limit & Upper Limit & $p$ value \\
\hline DRB $1 * 01$ & 1 & 35 & 3 & 4 & 44 & 8 & 0.314 & 0.034 & 2.94 & 0.386 \\
\hline DRB1*03 & 7 & 29 & 19 & 8 & 40 & 17 & 1.207 & 0.393 & 3.704 & 0.779 \\
\hline DRB1*04 & 5 & 31 & 14 & 5 & 43 & 10 & 1.387 & 0.37 & 5.207 & 0.738 \\
\hline DRB1*07 & 2 & 34 & 6 & 1 & 47 & 2 & 2.765 & 0.241 & 31.741 & 0.574 \\
\hline DRB1*10 & 1 & 35 & 3 & 1 & 47 & 2 & 1.343 & 0.081 & 22.219 & 1 \\
\hline DRB1*11 & 10 & 26 & 28 & 4 & 44 & 8 & 4.231 & 1.204 & 14.868 & 0.036 \\
\hline DRB1*12 & 0 & 36 & 0 & 1 & 47 & 2 & $\mathrm{NE}$ & $\mathrm{NE}$ & $\mathrm{NE}$ & 1 \\
\hline DRB1*13 & 3 & 33 & 8 & 4 & 44 & 8 & 1 & 0.209 & 4.776 & 1 \\
\hline DRB1*14 & 1 & 35 & 3 & 4 & 44 & 8 & 0.314 & 0.034 & 2.94 & 0.386 \\
\hline DRB1*15 & 6 & 30 & 17 & 8 & 40 & 17 & 1 & 0.314 & 3.188 & 1 \\
\hline DRB1*16 & 0 & 36 & 0 & 8 & 40 & 17 & $\mathrm{NE}$ & $\mathrm{NE}$ & $\mathrm{NE}$ & $0.009^{*}$ \\
\hline
\end{tabular}

Table 7: HLA-DRB1* frequencies and comparisons between patients with pediatric-onset $(\mathrm{N}=18)$ and adolescent-onset multiple sclerosis $(\mathrm{N}=23)$.

\begin{tabular}{|c|c|c|c|c|c|c|c|c|c|c|}
\hline \multirow{2}{*}{ HLA-DRB1* } & \multicolumn{3}{|c|}{$\begin{array}{l}\text { Adolescent-onset Multiple Sclerosis (48 } \\
\text { alleles) }\end{array}$} & \multicolumn{3}{|c|}{$\begin{array}{l}\text { Adult-Onset Multiple Sclerosis (246 } \\
\text { alleles) }\end{array}$} & \multicolumn{4}{|c|}{ Fisher Exact Test } \\
\hline & Positive & Negative & $\%$ & Positive & Negative & $\%$ & OR & Lower Limit & Upper Limit & $p$ value \\
\hline DRB1 ${ }^{*} 01$ & 4 & 44 & 8 & 19 & 227 & 7 & 1.086 & 0.352 & 3.347 & 0.776 \\
\hline $\mathrm{DRB} 1{ }^{*} 03$ & 8 & 40 & 17 & 22 & 224 & 9 & 2.036 & 0.848 & 4.892 & 0.119 \\
\hline DRB $1 * 04$ & 5 & 43 & 10 & 31 & 215 & 13 & 0.806 & 0.297 & 2.192 & 0.812 \\
\hline DRB1*07 & 1 & 47 & 2 & 10 & 236 & 4 & 0.502 & 0.063 & 4.017 & 1 \\
\hline DRB1*10 & 1 & 47 & 2 & 7 & 239 & 3 & 0.726 & 0.087 & 6.043 & 1 \\
\hline DRB1*11 & 4 & 44 & 8 & 42 & 204 & 17 & 0.442 & 0.151 & 1.295 & 0.19 \\
\hline DRB1*12 & 1 & 47 & 2 & 6 & 240 & 2 & 0.851 & 0.1 & 7.233 & 1 \\
\hline DRB1*13 & 4 & 44 & 8 & 16 & 230 & 7 & 1.307 & 0.417 & 4.095 & 0.753 \\
\hline DRB1*14 & 4 & 44 & 8 & 12 & 234 & 5 & 1.773 & 0.547 & 5.749 & 0.308 \\
\hline DRB1*15 & 8 & 40 & 17 & 41 & 205 & 17 & 1 & 0.436 & 2.293 & 1 \\
\hline DRB1*16 & 8 & 40 & 17 & 40 & 206 & 16 & 1.03 & 0.449 & 2.365 & 1 \\
\hline
\end{tabular}

Table 8: HLA-DRB1* frequencies and comparisons between patients with adolescent-onset $(\mathrm{N}=24)$ and adult-onset multiple sclerosis $(\mathrm{N}=123)$.

The allele frequencies of HLA-DRB1* in patients with early- and adult-onset disease are shown in Table 5. Two-sample chi-square revealed that the $\mathrm{DRB} 1{ }^{\star} 03$ allele was more frequent in patients with early disease onset $[\mathrm{OR}=2.213,(1.089-4.5), \mathrm{p}=0.043)$ but did not reach statistical significance according to the B-Y criterion. A tendency for lower DRB1*16 allele frequency was also noted in the early-onset group.

The allele frequencies of HLA-DRB1 ${ }^{*}$ in patients with pediatric disease onset and adult-onset MS are shown in Table 6. Patients with adult-onset disease onset had a significant increased frequency of $\mathrm{DRB} 1^{\star} 16$ allele versus patients with pediatric onset $(\mathrm{p}=0.004)$. The 
latter also showed a non-statistical significant tendency for higher $\mathrm{DRB}^{\star} 03$ and $\mathrm{DRB} 1^{\star} 11$ allele frequencies. Patients with adolescentonset MS showed increased frequency of the DRB1* 16 allele than the pediatric group $(\mathrm{p}=0.009)$ and a non-statistical significant tendency for lower DRB1*11 allele (Table 7). However, the adolescent group showed non-significant difference with the adult group, although a tendency for higher $\mathrm{DRB} 1^{\star} 03$ allele frequency and lower $\mathrm{DRB} 1^{\star} 11$ allele frequency was noted (Table 8 ).

\section{Discussion}

To our knowledge, this is the first study aiming at revealing the HLA-DRB1* allelic distribution in early-onset (both pediatric and adolescent) and adult-onset MS, in the Hellenic population. Our findings are in part new and in part standard, concerning previous results of HLA frequencies in Hellenic MS patients and HLAgenotyping in various other populations.

Our study has showed that the DRB1 ${ }^{\star} 15$ allele was significantly more frequent in MS patients than healthy controls, irrespective of age at disease onset. A protective effect of HLA-DRB1*11 allele was warranted, since MS patients showed decreased frequencies of this allele, irrespective of age at disease onset. Also, a protective effect of the DRB $1^{*} 16$ allele can be deduced by the lower frequency of this allele in the pediatric-onset group. A tendency for higher $\mathrm{DRB}^{\star} 03$ allele frequency was also noted for MS patients with early-onset disease onset versus healthy controls. In respect of MS age at onset, the $\mathrm{DRB}^{\star}{ }^{\star} 16$ allele was found less prevalent in the pediatric group, than the adolescent-onset and adult-onset group, with a tendency of all MS patients with early-onset disease to present with lower $\mathrm{DRB} 1^{\star} 16$ allele frequencies. All patients with early-onset MS had a tendency for higher $\mathrm{DRB} 1^{\star} 03$ frequency versus the adult-onset group, while the pediatric-onset group had a non-significant higher frequency of $\mathrm{DRB}^{\star} 11$ allele than the adolescent-onset group.

Our results are consistent with previous studies showing greater susceptibility to MS in patients carrying the HLA-DRB1* 15 allele [4-8]. Also, the protective effect of the HLA-DRB1* 11 allele has been also confirmed in previous studies [30,31]. However, our study did not reproduce the association between the presence of HLA-DRB1 ${ }^{\star} 15$ and earlier age of MS onset $[18,19]$. We have detected two studies with similar negative findings concerning early-onset MS [32,33]. One possible explanation for this discrepancy could be the parent of origin effect on age of MS onset and DRB1* 15 . In specific, one study has showed that maternally transmitted $\mathrm{DRB1} 1^{\star} 15$ promotes a lower age of MS onset [34]. In our research, we have no information about the genotypes of the patients' parents. A second reason could be that given the association between vitamin D levels and DRB1 ${ }^{\star} 15$ expression, we hypothesize that differences in MS age at onset according to the presence of $\mathrm{DRB} 1^{\star} 15$ are more prominent in populations with low or graded sun exposure [10]. Since in Hellas sun exposure is particularly high throughout the year, expression of $\mathrm{DRB}^{\star} 15$ could be more homogenously distributed among the population and confers no risk for earlier disease onset, or larger samples are needed to detect HLAalleles frequency discrepancies, if they truly exist.

The protective role of $\mathrm{DRB} 1{ }^{\star} 16$ for early onset of the disease was, to our knowledge, a new finding that must be verified in future studies. However, this finding must be interpreted with caution since DRB $1^{\star} 16$ was totally absent in the pediatric group with only 18 patients examined (36 alleles), while no statistical differences or tendencies for this allele were noted between the adolescent-onset group and the adult-onset group. Interestingly, X-linked adrenoleukodystrophy, which occurs during childhood and resembles MS, has been associated with DRB1*16 [35].

Interestingly, $\mathrm{DRB1} 1^{\star} 03$ allele was more prevalent in early-onset MS, however, the difference did not reach statistical significance according to our B-Y statistical criterion for $p$ value correction. This allele has been previously associated with MS susceptibility and with the presence of Ig Moligoclonal band within the cerebrospinal fluid of these patients [36]. In a meta-analysis $D R B 1^{\star} 03$ was identified as "probably the only risk factor for MS besides $\mathrm{DRB}^{\star} 15$ and a common genetic foundation for autoimmune disease" at least for adult-onset MS [37]. DRB $1^{\star} 03$ allele does not seem to affect clinical disease severity, cognition or cerebral atrophy, although in one study it was associated with better MS prognosis [38]. Interestingly, DRB1 ${ }^{*} 03$ allele has been strongly associated with neuromyelitis optica (NMO), a humoral-mediated autoimmune disease entity associated with the presence of anti-aquaporin antibodies [39]. According to these findings of the presumed better MS prognosis and NMO association, the relation of $\mathrm{DRB1} 1^{\star} 03$ allele with early-onset MS could likely account for different pathogenetic mechanisms in this group of patients, most probably favoring humoral rather than cellular immune responses. In a certain population with certain genetic/immunogenetic background, the difference of HLA-distribution among age groups shows that the proposed immune differentiation for early-onset MS could be attributed to the diverse sex hormone profile during this ageperiod, which warrants further study by future animal and/or human research.

We have to admit that the sample size of MS patients with age at onset below 18 years old is low, thus decreasing the power of the study. Although, we could argue that early-onset MS consists only the 3-5\% of MS patients, thus our sample is satisfactory representative of the MS population [17]. However, for $\mathrm{p}$ value correction, we have adopted a less conservative method than the Bonferroni, thus minimizing type II error. Finally, due to our small sample we have not examined epistatic mechanisms in this study.

In conclusion, our findings demonstrate the role of HLA-DRB1* in MS susceptibility/protectivity and age at onset. New findings in this study are the putative predisposing role of $\mathrm{DRB} 1^{\star} 03$ allele and the protective role of the $\mathrm{DRB} 1^{\star} 16$ allele for early-onset MS. It is necessary that our findings would be confirmed by future studies, especially in Hellas, where evidence is scarce. Studies in different countries, could further elucidate the interplay between environmental (e.g. sun exposure) and genetic factors in MS. To our opinion, delineating the role of HLA in MS could foster future individualized prognostic making and therapeutic choices that will change disease course and optimize quality of life of MS patients.

\section{Conflict of Interest Statement}

M. Anagnostouli has received research support and honoraria from Novartis Pharma, and research support from Merck Serono, Biogen Idec, Bayer Schering Pharma, UCB and Teva Pharmaceutical Industries Ltd.

\section{References}

1. Ramagopalan SV, Dyment DA, Ebers GC (2008) Genetic epidemiology: the use of old and new tools for multiple sclerosis. Trends Neurosci 31: 645-652. 
Citation: Anagnostouli MC, Manouseli A, Artemiadis AK, Katsavos S, Fillipopoulou C et al. (2014) HLA-DRB1* Allele Frequencies in Pediatric, Adolescent and Adult-Onset Multiple Sclerosis Patients, in a Hellenic Sample. Evidence for New and Established Associations. J Mult Scler 1: 104. doi:10.4172/jmso.1000104

Page 8 of 8

2. Katsavos S, Anagnostouli M (2013) Biomarkers in Multiple Sclerosis: An Up-to-Date Overview. MultSclerInt 2013: 340508.

3. Naito S, Namerow N, Mickey MR, Terasaki PI (1972) Multiple sclerosis: association with HL-A3. Tissue Antigens 2: 1-4.

4. Jersild C, Fog T, Hansen GS, Thomsen M, Svejgaard A, et al. (1973) Histocompatibility determinants in multiple sclerosis, with special reference to clinical course. Lancet 2: 1221-1225.

5. International Multiple Sclerosis Genetics Consortium; Wellcome Trust Case Control Consortium 2, Sawcer S, Hellenthal G, Pirinen M, Spencer CC, et al. (2011) Genetic risk and a primary role for cell-mediated immune mechanisms in multiple sclerosis. Nature 476: 214-219.

6. Ito H, Yamasaki K, Kawano Y, Horiuchi I, Yun C, et al. (1998) HLA-DPassociated susceptibility to the optico-spinal form of multiple sclerosis in the Japanese. Tissue Antigens 52: 179-182.

7. Quelvennec E, Bera O, Cabre P, Alizadeh M, Smadja D, et al. (2003) Genetic and functional studies in multiple sclerosis patients from Martinique attest for a specific and direct role of the HLA-DR locus in the syndrome. Tissue Antigens 61: 166-171.

8. Aláez C, Corona T, Ruano L, Flores H, Loyola M, et al. (2005) Mediterranean and Amerindian MHC class II alleles are associated with multiple sclerosis in Mexicans. ActaNeurolScand 112: 317-322.

9. Balnyte R, Rastenyte D, Vaitkus A, Mickeviciene D, Skrodeniene E, et al (2013) The importance of HLA DRB1 gene allele to clinical features and disability in patients with multiple sclerosis in Lithuania. BMC Neurol 13: 77 .

10. Ramagopalan SV, Maugeri NJ, Handunnetthi L, Lincoln MR, Orton SM, et al. (2009) Expression of the multiple sclerosis-associated MHC class II Allele HLA-DRB1 ${ }^{\star} 1501$ is regulated by vitamin D. PLoS Genet 5: e1000369.

11. DeLuca GC, Alterman R, Martin JL, Mittal A, Blundell S, et al. (2013) Casting light on multiple sclerosis heterogeneity: the role of HLA-DRB1 on spinal cord pathology. Brain 136: 1025-1034.

12. Leone MA, Barizzone N, Esposito F, Lucenti A, Harbo HF, et al. (2013) Association of genetic markers with CSF oligoclonal bands in multiple sclerosis patients. PLoS One 8: e64408.

13. Waubant E, Mowry EM, Krupp L, Chitnis T, Yeh EA, et al. (2013) Antibody response to common viruses and human leukocyte antigenDRB1 in pediatric multiple sclerosis. MultScler 19: 891-895.

14. Irizar H, Muñoz-Culla M, Zuriarrain O, Goyenechea E, Castillo-Triviño T, et al. (2012) HLA-DRB1 ${ }^{\star} 15: 01$ and multiple sclerosis: a female association? MultScler 18: 569-577.

15. Anagnostouli M, Anagnostoulis G2, Katsavos S3, Panagiotou M4, Kararizou E4, et al. (2014) HLA-DRB1 15:01 and Epstein-Barr virus in a multiple sclerosis patient with psoriasis, nasopharyngeal and breast cancers. Lessons for possible hidden links for autoimmunity and cancer. J NeurolSci 339: 26-31.

16. Dyment DA, Herrera BM, Cader MZ, Willer CJ, Lincoln MR, et al. (2005) Complex interactions among MHC haplotypes in multiple sclerosis: susceptibility and resistance. Hum Mol Genet 14: 2019-2026.

17. Gadoth N (2003) Multiple sclerosis in children. Brain Dev 25: 229-232.

18. Disanto G, Magalhaes S, Handel AE, Morrison KM, Sadovnick AD, et al. (2011) HLA-DRB1 confers increased risk of pediatric-onset MS in children with acquired demyelination. Neurology 76: 781-786.

19. Smestad C, Brynedal B, Jonasdottir G, Lorentzen AR, Masterman T, et al (2007) The impact of HLA-A and -DRB1 on age at onset, disease course and severity in Scandinavian multiple sclerosis patients. Eur J Neurol 14: 835-840.

20. Wu JS, Qiu W, Castley A, James I, Mastaglia FL, et al. (2010) Modifying effects of HLA-DRB1 allele interactions on age at onset of multiple sclerosis in Western Australia. MultScler 16: 15-20.

21. Oh HH, Kwon SH, Kim CW, Choe BH, Ko CW, et al. (2004) Molecular analysis of HLA class II-associated susceptibility to neuroinflammatory diseases in Korean children. J Korean Med Sci 19: 426-430.
22. Spence RD, Voskuhl RR (2012) Neuroprotective effects of estrogens and androgens in CNS inflammation and neurodegeneration. Front Neuroendocrinol 33: 105-115.

23. Van Lambalgen R, Sanders EA, D'Amaro J (1986) Sex distribution, age of onset and HLA profiles in two types of multiple sclerosis. A role for sex hormones and microbial infections in the development of autoimmunity? J NeurolSci 76: 13-21.

24. Kouri I, Papakonstantinou S, Bempes V, Vasiliadis HS, Kyritsis AP, et al. (2011) HLA associations with multiple sclerosis in Greece. J NeurolSci 308: 28-31.

25. Bozikas VP, Anagnostouli MC, Petrikis P, Sitzoglou C, Phokas C, et al. (2003) Familial bipolar disorder and multiple sclerosis: a threegeneration HLA family study. ProgNeuropsychopharmacol Biol Psychiatry 27: 835-839.

26. McDonald WI, Compston A, Edan G, Goodkin D, Hartung HP, et al (2001) Recommended diagnostic criteria for multiple sclerosis: guidelines from the International Panel on the diagnosis of multiple sclerosis. Ann Neurol 50: 121-127.

27. Marsh SG (2012) Nomenclature for factors of the HLA system, update June 2012. Tissue Antigens 80: 289-293.

28. Cros P, Allibert P, Mandrand B, Tiercy JM, Mach B (1992) Oligonucleotide genotyping of HLA polymorphism on microtitre plates. Lancet 340: 870-873.

29. Benjamini Y, Drai D, Elmer G, Kafkafi N, Golani I (2001) Controlling the false discovery rate in behavior genetics research. Behav Brain Res 125: 279-284.

30. Dean G, Yeo TW, Goris A, Taylor CJ, Goodman RS, et al. (2008) HLADRB1 and multiple sclerosis in Malta. Neurology 70: 101-105.

31. Ramagopalan SV, Morris AP, Dyment DA, Herrera BM, DeLuca GC, et al. (2007) The inheritance of resistance alleles in multiple sclerosis. PLoS Genet 3: 1607-1613.

32. Boiko AN, Gusev EI, Sudomoina MA, Alekseenkov AD, Kulakova OG, et al. (2002) Association and linkage of juvenile MS with HLA-DR2(15) in Russians. Neurology 58: 658-660.

33. Barcellos LF, Sawcer S, Ramsay PP, Baranzini SE, Thomson G, et al. (2006) Heterogeneity at the HLA-DRB1 locus and risk for multiple sclerosis. Hum Mol Genet 15: 2813-2824.

34. Ramagopalan SV, Byrnes JK, Dyment DA, Guimond C, Handunnetthi L, et al. (2009) Parent-of-origin of HLA-DRB1 ${ }^{\star} 1501$ and age of onset of multiple sclerosis. J Hum Genet 54: 547-549.

35. Berger J, Bernheimer H, Faé I, Braun A, Roscher A, et al. (1995) Association of X-linked adrenoleukodystrophy with HLA DRB1 alleles. BiochemBiophys Res Commun 216: 447-451.

36. de la Concha EG, Cavanillas ML, Cénit MC, Urcelay E, Arroyo R, et al. (2012) DRB1 ${ }^{\star} 03: 01$ haplotypes: differential contribution to multiple sclerosis risk and specific association with the presence of intrathecalIgM bands. PLoS One 7: e31018.

37. Zhang Q, Lin CY, Dong Q, Wang J, Wang W (2011) Relationship between HLA-DRB1 polymorphism and susceptibility or resistance to multiple sclerosis in Caucasians: a meta-analysis of non-family-based studies. Autoimmun Rev 10: 474-481.

38. Van der Walt A, Stankovich J, Bahlo M, Taylor BV, Van der Mei IA, et al. (2011) Heterogeneity at the HLA-DRB1 allelic variation locus does not influence multiple sclerosis disease severity, brain atrophy or cognition. MultScler 17: 344-352.

39. Brum DG, Barreira AA, dos Santos AC, Kaimen-Maciel DR, Matiello M, et al. (2010) HLA-DRB association in neuromyelitisoptica is different from that observed in multiple sclerosis. MultScler 16: 21-29. 